to retrospectively report antisocial behaviour in childhood (OR $2.38,95 \%$ CI 1.04 to 5.45). Increased odds of delayed onset PTSD were seen for those with poor physical health at both phases 1 and 2 (OR 4.29, 95\% CI 1.44 to 12.77) and for those with CMD onset between phases 1 and 2 (OR 4.80, 95\% CI 2.16 to 10.67).

Conclusions The prevalence of delayed onset PTSD in this study was low; however, those most at risk were military personnel in lower ranks, with poorer physical health. Leaving the military was not associated with delayed onset PTSD. Delayed onset PTSD appears to parallel the onset of other psychological health problems.

\section{P117 PREVALENCE AND PREDICTORS OF DELAYED ONSET PTSD IN MILITARY PERSONNEL: IS THERE EVIDENCE FOR THIS DISORDER? RESULTS OF A PROSPECTIVE UK COHORT STUDY}

Laura Goodwin, Margaret Jones, Josefin Sundin, Simon Wessely, Roberto J Rona, Nicola T Fear King's College, London, UK

10.1136/oemed-2011-100382.331

Objectives Delayed onset posttraumatic stress disorder (PTSD) occurs 6 months or more after a traumatic event. Onset may occur after individuals have left the military, when they no longer have access to military occupational support. This study will investigate prospective predictors for this disorder, to identify those most at risk of delayed onset.

Methods 1397 participants from a two phase prospective cohort study in U.K. military personnel. Delayed onset PTSD was categorised as participants who did not meet the diagnostic criteria for PTSD at phase 1, who met the criteria by phase 2 . Socio-demographic and military characteristics were assessed at both phases, in addition to childhood adversity, physical health status, common mental disorder (CMD), alcohol misuse and multiple physical symptoms.

Results Three percent $(\mathrm{N}=44)$ met the criteria for delayed onset PTSD. Participants with delayed onset PTSD were less likely to be officers (OR $0.20,95 \%$ CI 0.04 to 0.95 ), and were more likely 